\title{
A Ubiquitous Real-Time Motion Artifact Rejection technique for Remote NIBP Monitoring of Hypertensive Patients
}

\author{
Ali Hasnain ${ }^{1}$, Mudaser Awan ${ }^{1}$ and Muddassar Farooq ${ }^{1}$ \\ ${ }^{1}$ Next Generation Intelligent Networks Research Center (nexGIN RC), \\ National University of Computer and Emerging Sciences (FAST-NUCES), \\ Islamabad 44000, Pakistan
}

\begin{abstract}
The periodic and frequent measurements of blood pressure for hypertensive patients are must to do their effective and efficient monitoring. Existing non-invasive blood pressure measurement instruments mandate a relaxed posture - it is considered a prerequisite to factor out motion artifacts in the reading - before taking the final reading. This requirement causes not only a great discomfort for the patients but also renders the equipment useless in providing ubiquitous and real-time onthe-move remote monitoring of the patients. In this paper, we propose a novel technique to get accurate measurement of the blood pressure while walking with the free end motion of the arm. The approach utilizes an accelerometer mounted on the cuff wrapped over the upper arm in order to determine the noise superimposed over the oscillometric signal of the patient's blood pressure. The system is tested on a mannequin's limb simulated to have arm movements with the oscillometric signal. The results of our analysis show that the system is robust in determining the correct blood pressure irrespective of the noise level produced due to the physical movement of the arm. We believe that our system will become a cardinal component of a cost-efficient, highly-reliable ubiquitous remote patient monitoring system.
\end{abstract}

Keywords - Non-Invasive Blood Pressure, Oscillometric Signal, Accelerometer, Simulator.

\section{INTRODUCTION}

Hypertension is one of the most common cardiovascular diseases - it is known to affect the lives of 7 million people throughout the world - that contributes to a total of $13 \%$ global mortality [1]. The latter stages of hypertension in antenatal patients - pre-eclampsia, eclampsia and pregnancy-induced hypertension - significantly contributed to the above-mentioned alarming mortality statistics. In this context, we emphasize the need of developing systems that can accurately monitor in real-time the blood pressure (BP) of a patient to manage him in an efficient and effective manner. The patients, having being diagnosed as hypertensive, are given a clinical advise by medical experts to periodically measure their blood pressure several times a day [2]. As a result, the patients - most of them belong to this category
- having a busy and hectic professional schedule mostly ignore the advice to periodically measure their BP because of the need to be in a relaxed posture for 10-15 minutes before taking the measurement.

To this end, we propose a novel device for non-invasive blood pressure (NIBP) monitoring system - the patients can wear it on their arms while doing their routine activities that has the capability to automatically measure BP ubiquitously at periodic intervals - recommended by the physicians - without the need for patients to be in a relaxed posture for certain amount of time. We suggest that the instrument be worn on the cuff; therefore, we only model the movement of arm and its artifacts on measurements of the BP samples. We believe that such a system will become a cardinal component of a remote patient monitoring system ${ }^{1}$ for the hypertensive patients, which has the ability to measure in real-time, onthe-move BP - a feature that would greatly benefit busy professionals - in an effective and cost-efficient manner.

In order to show the efficacy of our proposed technique, we have developed a simulator that can measure the motion artifacts in measurements of blood pressure during walk. The novel dimension of our work is the use of accelerometer to estimate the noise and filter it from the oscillometric signal. Oscillometric signal represents the pressure oscillations produced while deflating the cuff from supra-systolic pressure to sub-systolic pressure after occlusion of blood flow in the extremities. The normal frequency range of the noise generated due to an arm swing is $0-2 \mathrm{~Hz}$ and it also overlaps the bandwidth of the oscillometric signal.

To the best of our knowledge, the existing techniques use conventional filters such - Kalman filter and pulse matching algorithm - to remove the motion artifacts during the blood pressure measurement [3]. The use of such techniques, however, is not appropriate due to a significant overlap in the noise frequencies and the oscillometric signal. The problem of motion artifacts compensation, thus, poses a unique challenge to the conventional blood pressure monitoring techniques. In comparison, we use an accelerometer as a feedback

\footnotetext{
${ }^{1}$ This research is the part of Remote Patient Monitoring System project. http://rpms.nexginrc.org/
} 


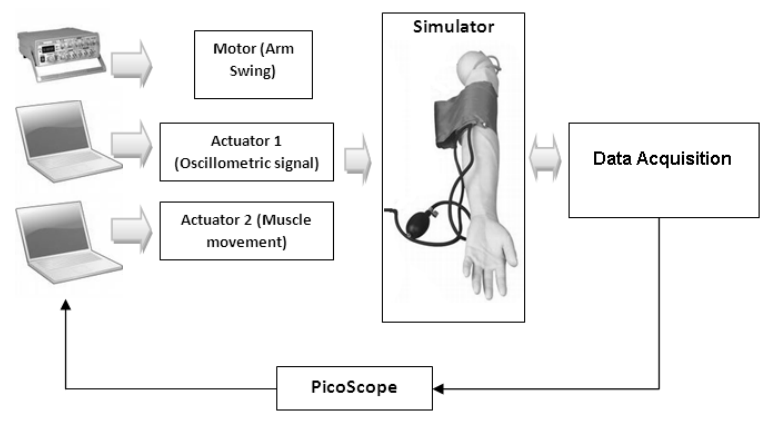

Fig. 1: System Block Diagram

to remove the motion artifacts using an adaptive filters.

The rest of the paper is organized as follows. In Section 2 , we provide a brief overview of the related work. In Section 3, we describe the conceptual and block diagram of our simulator. In Section 4 we first demonstrate, in detail, the effect of motion artifacts on the blood pressure signal and then present our technique to remove it. We also discuss the results in Section 4 and finally conclude the paper in Section 5 with an outlook to our future research.

\section{RELATED WORK}

Charbonnier et al. made use of accelerometer in 24-h ambulatory systolic blood pressure (ASBP) recording [4], but they used accelerometer to only measure the intensity of the physical activity to conclude whether the body is static or dynamic. Yoonseo et al. have studied the rejection of motion artifacts in blood pressure measurements while patient is transported to a hospital in an ambulance [3].

\section{ARCHITECTURE}

\section{A. Theory}

Blood pressure (BP) measurements are disturbed mainly because of movement, breathing and talking [5]. We have focused on removing the errors due to the motion artifacts - we consider walking as the primary movement source - that are responsible for errors in the measured blood pressure sample. Even though arms are not the direct means of locomotion, but they do play a vital role in maintaining stability of human body during locomotion. Researchers have reported a fixed relationship between the arm and leg movements: $1 / 1,2 / 1$, $3 / 1,4 / 1$ or $5 / 1$ [6]. In our simulator, the speed of movement of arms is computed by using a simple relationship derived from arm and leg movements [6]:

$$
y=1.67 x
$$

where, $y$ is the frequency of arm and $x$ is the frequency of leg.

The expansion and contraction of the biceps/triceps muscles are mainly responsible for the induced motion artifacts during the measurement of the blood pressure. Therefore, we generate two signals in our blood pressure simulator to model the muscle behavior: (1) Oscillometric signal (frequency 1-4 $\mathrm{Hz}$ ), and (2) Muscles movement (frequency $0-2 \mathrm{~Hz}$ )

\section{B. Simulator Design}

Our simulator consists of a mannequin's left limb which is pivoted on humeral joint with the armature of a DC motor. A CONE cuff of an adult's size $(24-36 \mathrm{~cm})$ is worn over the main artery (2-3 cm above the elbow joint). Two central locking actuators, with a cord around the inflated cuff, are used to simulate the oscillometric and muscles movement signals. The muscle movement simulations are produced by contracting and relaxing the BP cuff by the cord using the actuator and applying pressure on the cuff corresponding to the contraction and relaxation of bicep/tricep muscles. Similarly, oscillometric signal is generated using another actuator. Both actuators work simultaneously to simulate the heart beat (HB) and muscle movement (MM). We have used Motorola MPX2050GP pressure transducer that is cascaded with a signal conditioning circuitry to measure even minor pressure changes because of the linear motion of the actuator.

To simulate the effect of arms movement on the measured blood pressure value, an artificial blood pressure simulator is created that simply logs the output of pressure transducer data from the cuff in a computer. The logged data is analyzed to cater for errors induced due to the movement.

\section{Data Acquisition}

Feedback: The feedback of arm movement is acquired using 3 axis accelerometer (LIS3L02AS4) which gives acceleration in each axis corresponding to the movement of arm. The accelerometer gives an analog output signal for each axis, which is amplified using operational amplifier and fed to an 8-bit Analog to Digital Convertor (ADC0832) that ultimately delivers the data to the micro-controller (AT89C8051).

BP Measurement. At the same time, the data from the blood pressure cuff is measured in terms of changes in the pressure induced due to HB, MM, and arm-swing movement. The pressure transducer gives differential voltage output corresponding to the change in the pressure of cuff. The value is amplified using Instrumentation Amplifier (AD620), and is then given to an Active Band Pass Filter (BPF) with a band pass region from $1 \mathrm{~Hz}$ to $4 \mathrm{~Hz}$ that actually removes the noise 


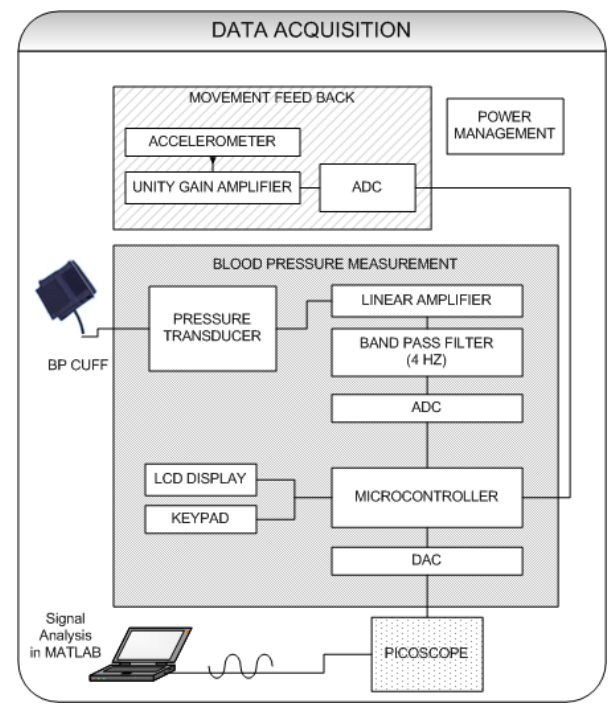

Fig. 2: Data Acquisition Block Diagram

induced due to switching currents. The output of BPF is fed to an analog to digital converter (ADC), which converts the ana$\log$ waveforms, obtained from the pressure transducer, into digital so that it can be processed by the micro-controller. The micro-controller follows a pulsation algorithm to compute the Systolic and Diastolic BP values based on the data acquired from the ADC.

\section{EXPERIMENTS AND RESULTS}

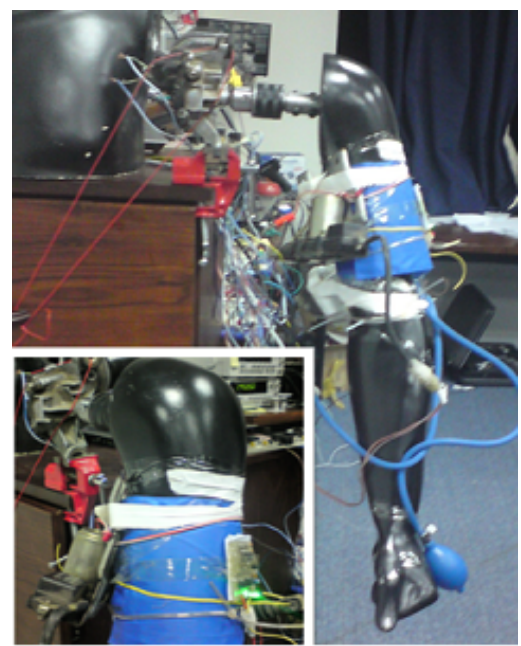

Fig. 3: Blood Pressure Simulator of a walking person

Data of five adult healthy subjects is logged in the PC through single channel PC Oscilloscope (PicoScope 2105).
The logged data is normalized and converted into an audio (.wav) file format in MATLAB. This wave file is played on PC audio output port and given to an amplifier module which converts the audio signal profile to an equivalent mechanical actuator movements which simulate HB. We have used Equation (1) to calculate the arm's swing frequency by taking the test condition of $4 \mathrm{~km} / \mathrm{hr}$ walking speed. The data from the accelerometer and pressure transducer is sent to the micro-controller which combines the data and gives an equivalent wave-form on Digital to Analog convertor (DAC) output. This output is recorded using PicoScope and imported in MATLAB for analysis. The overall simulator system is shown in the Figure 3.

The data from artificially induced pulsations is logged in the PC system from the pressure transducer that is attached to the cuff. To remove the motion artifacts, following testing scenarios are generated and the data is logged for each one of them in PC (see Figure 4):

- Data of Oscillometric signal

- Data of Muscle movement

- Accelerometer signal (Arm swing)

- Blood Pressure with Muscle movement

The Heart Beat is the simulated waveform of a heartbeat without any errors induced due to the movement. It has a sinusoidal shape with frequency of $2.7 \mathrm{~Hz}$. The Muscle Movement waveform shows simulated contraction and relaxation of biceps/triceps that normally occur during walk. The Heart Beat + Muscle Movement waveform is the distorted waveform obtained as a cumulative response of an arm-swing, heartbeat and muscle movement. The accelerometer waveform is only due to an arm-swing without heartbeat and muscle movement.

To remove the errors induced because of movements, each signal is sampled at a sampling frequency of $100 \mathrm{~Hz}$ for 5 seconds. We used curve fitting to determine an equation for each signal. The equation for heart beat signal with a muscle movement is:

$$
\begin{array}{r}
y=a 1 * \sin (b 1 * x+c 1)+a 2 * \sin (b 2 * x+c 2) \\
+a 3 * \sin (b 3 * x+c 3)
\end{array}
$$

Coefficients (with 95\% confidence bounds):

$a 1=2.505(-1.347,6.357)$

$b 1=0.0002673(-0.005072,0.005607)$

$c 1=1.221(-2.85,5.293)$

$a 2=4.138(3.886,4.391)$

$b 2=0.1729(0.1724,0.1733)$

$c 2=0.5441(0.4477,0.6405)$

$a 3=0.07745(-0.1733,0.3282)$ 


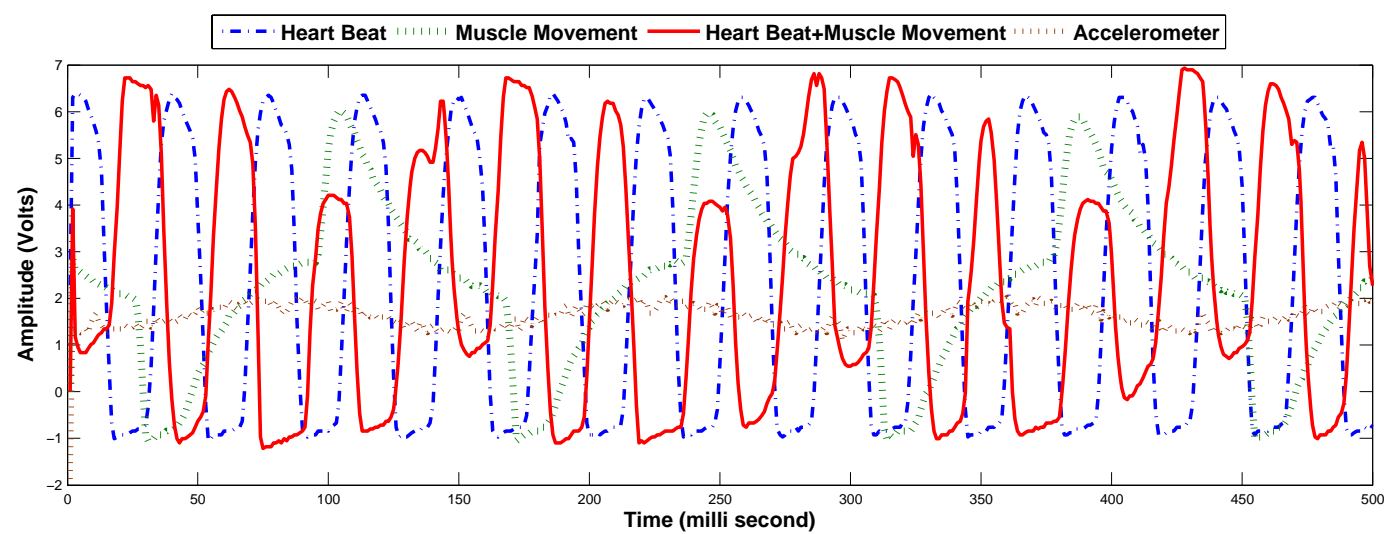

Fig. 4: Waveform for Heartbeat, Muscle Movement, Heartbeat with Muscle Movement and Arm Swing

$b 3=0.1631(0.1397,0.1865)$

$c 3=2.618(-2.58,7.817)$

The noise is removed from the blood pressure cuff signal by using an FIR-Least Mean Squares (FIR-LMS) adaptive filter that also takes into account the feedback from the output of the accelerometer. From observation one can see the relationship between arm swing and errors induced in blood pressure results. The goal is to clearly identify the peaks of the true signal in order to determine the correct BP. Figure 5 shows the results before and after applying the adaptive filter.

\section{CONCLUSION}

In this study, we have presented a novel technique that filters ourt the motion artifices in the measured samples of blood pressure. We have validated our technique in a customized NIBP simulator, which artificially induces noise due to humeral joint and biceps/triceps movement. The resulting motion artifact compensator is designed with the help of an inertial sensor. The output of the accelerometer is fed to an adaptive filter which eliminates the motion artifacts from the noisy oscillometric signal. We have used a simple and cost-efficient technique - once we compare it with existing expensive BP simulators - to generate the blood pressure data. We believe that our proposed system will become a cardinal component of remotely monitoring hypertensive patients while they are engaged in their routine activities. The correct data can be ubiquitously transferred using a mobile network - to a tertiary care hospital.

In near future, we would like to extend our work by using $2 \mathrm{D}$ and $3 \mathrm{D}$ accelerometers. We also want to evaluate our ap- proach to remove other noise artifacts due to breathing and talking.

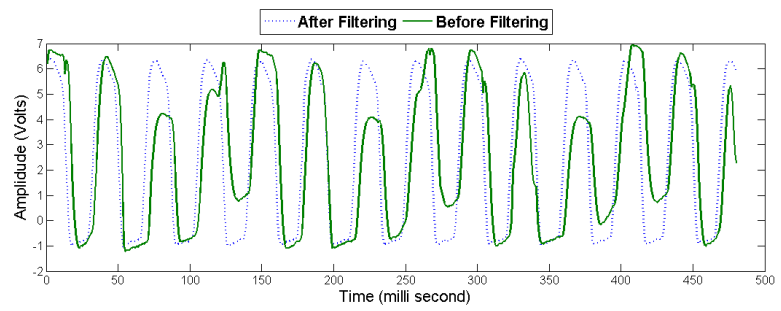

Fig. 5: Oscillometric signal before and after filtering

\section{REFERENCES}

1. WHO report, "Regional consultation on hypertension prevention and control", United Arab Emirates, 2003.

2. G.R. Jeon, J.H. Jung, I.C. Kim, A.Y. Jeon, S.H. Yoon, J.M. Son, J.H. Kim, S.Y. Ye, J.H. Ro, D.H. Kim, C.H. Kim, "A Simulation for Estimation of the Blood Pressure using Arterial Pressure-volume Model", Proceedings of World Academy of Science, Engineering and Technology, Volume 24, pp. 366-371, 2007.

3. Y. Koo, J. Kang, H. Shin, M.Y. Jung, G.J. Suh, H.C. Kim ,"Preliminary Study of Motion Artifact Rejection for NIBP measurement in an Ambulance", Engineering in Medicine and Biology Society, pp. 705-708, 2007.

4. S. Charbonnier, J.P. Siche, J.M. Mallion, "Toward a portable blood pressure recorder device equipped with an accelerometer", Medical Engineering \& Physics, vol. 21, no. 5, pp. 343-352, 1999.

5. J.J. Lynch, J.M Long, S.A. Thomas, K.L. Malinow, A.H. Katcher, "The Effects of Talking on the Blood Pressure of Hypertensive and Normotensive Individuals", Psychosomatic Medicine, vol. 43, no. 1, pp. 25-33, 1981.

6. T. Wannier, C. Bastiaanse, G. Colombo, "Arm to Leg coordination in human during walking, creeping and swimming activities", Experimental Brain Research, vol. 141, no. 3, pp. 375-379, Springer, 2001. 